\title{
CHAPTER 64
}

\section{Brussels}

\author{
Daniel Röder
}

The MATSim scenario for Brussels was developed as part of the SustainCity project. This project's goal was to couple an urban land use model, in this case UrbanSim, with the MATSim mobility simulation, to evaluate transport policy impact on urban land use and vice versa. A detailed description of this coupling is given by Nicolai (2013) and others. A detailed description of the scenario development is found in Röder et al. (2013).

The scenario covered the greater Brussels area in Belgium; input data was derived from two main sources. The population was directly generated from the UrbanSim model, covering a total of 860214 persons. At home- and at work-locations (per person) were given and converted into a daily home-work-home plan. For computational reasons, a randomly-drawn population sample of one percent was used. OSM was sourced for the street network generation, which consisted of 10861 nodes and 19830 links, i.e., using mainly the trunk road network.

For the modeling of public transport, two different approaches were tested: first, the MATSim default approach for scenarios where no detailed transit schedule is available, based on either: beeline distance and average speed, or network-based freespeed travel times and a designated factor. The second approach was not part of the MATSim core during the project, but was available as a contribution (matrixBasedPtRouter, see Chapter 20). It was based on O-D travel time matrices between transit stops, i.e., travel times for all relations were computed in a pre-process. The travel times can based on a real-world-schedule or certain assumptions which can take spatial coverage into account. Advantages of this model are obvious; on one hand, it may depict spatial coverage with public transport supply-here, distance to the next transit stop influences travel time. On the other hand, it may depict the real network, i.e., routes and lines and possible waiting times for switching. Both approaches were compared against travel times and mode share measures from a SATURN (Simulation and Assignment of Traffic to Urban Road Networks) model. Since the matrix-based approach came closer to this model, further investigations were based on that.

\section{How to cite this book chapter:}

Röder, D. 2016. Brussels. In: Horni, A, Nagel, K and Axhausen, K W. (eds.) The Multi-Agent Transport Simulation MATSim, Pp. 405-406. London: Ubiquity Press. DOI: http://dx.doi.org/10.5334/baw.64. License: CC-BY 4.0 
To evaluate the model's sensitivity to certain policies, a cordon toll scenario was set up, where a toll is charged between 6 and 10 am every time a car passed a cordon border, i.e., every time a car entered a link crossing a cordon border defined by the Brussels freeway ring. Accessibility was calculated and compared for both scenarios. Röder et al. (2013) provides a detailed analysis. 